\title{
Log-concavity of $P$-recursive sequences
}

\author{
Qing-hu Hou and Guojie Li \\ School of Mathematics \\ Tianjin University \\ Tianjin 300072, China \\ qh_hou@tju.edu.cn, 1017233006@tju.edu.cn
}

\begin{abstract}
.
We consider the higher order Turán inequality and higher order logconcavity for sequences $\left\{a_{n}\right\}_{n \geq 0}$ such that

$$
\frac{a_{n-1} a_{n+1}}{a_{n}^{2}}=1+\sum_{i=1}^{m} \frac{r_{i}(\log n)}{n^{\alpha_{i}}}+o\left(\frac{1}{n^{\beta}}\right),
$$

where $m$ is a nonnegative integer, $\alpha_{i}$ are real numbers, $r_{i}(x)$ are rational functions of $x$ and

$$
0<\alpha_{1}<\alpha_{2}<\cdots<\alpha_{m}<\beta .
$$

We will give a sufficient condition on the higher order Turán inequality and the $\ell$-log-concavity for $n$ sufficiently large. Many $P$-recursive sequences fall in this frame. At last, we will give a method to find the $N$ such that for any $n>N$, the higher order Turán inequality holds.
\end{abstract}

Keywords: higher order Turán inequality, log-concave, $P$-recursive sequence, asymptotic estimation.

AMS Classifications: 41A60, 05A20, 41A58.

\section{Introduction}

The Turán inequalities and the higher order Turán inequalities arise in the study of Maclaurin coefficients of real entire functions in the Laguerre-Pólya class [15]. A sequence $\left\{a_{n}\right\}_{n \geq 0}$ of real numbers is said to satisfy the Turán inequalities or to be log-concave, if

$$
a_{n}^{2}-a_{n-1} a_{n+1} \geq 0, \quad \forall n \geq 1 .
$$

The Turán inequalities are also called Newton's inequality [12,17]. For more results on the log-concavity, we refer to [4,10, 13].

A sequence $\left\{a_{n}\right\}_{n \geq 0}$ is said to satisfy the higher order Turán inequalities if for all $n \geq 1$,

$$
4\left(a_{n}^{2}-a_{n-1} a_{n+1}\right)\left(a_{n+1}^{2}-a_{n} a_{n+2}\right)-\left(a_{n} a_{n+1}-a_{n-1} a_{n+2}\right)^{2} \geq 0 .
$$


Chen, Jia and Wang [2] use the Hardy-Ramanujan-Rademacher formula to prove that, when $n \geq 95$, the partition function satisfies the higher order Turán inequality. Dimitrov [5] observed that for a real entire function $\Psi(x)$ in the Laguerre-Pólya class, the Maclaurin coefficients satisfy the higher order Turán inequalities. Došlić [6] proved some combinatorial sequences are log-balanced. Wang [16] proved some combinatorial sequences satisfy the higher order Turán inequalities. Griffin, Ono, Rolen, and Zagier [7] give a straightforward way to check whether a given sequence eventually satisfies the higher Turán inequalities.

Let $\varphi$ be the operator given by

$$
\varphi\left\{a_{n}\right\}_{n \geq 0}=\left\{a_{n+1}^{2}-a_{n} a_{n+2}\right\}_{n \geq 0},
$$

and let $\varphi^{k}$ be the composition of $\varphi k$ times with itself. If

$$
\varphi\left\{a_{n}\right\}_{n \geq 0}, \quad \varphi^{2}\left\{a_{n}\right\}_{n \geq 0}, \quad \cdots, \quad \varphi^{r}\left\{a_{n}\right\}_{n \geq 0}
$$

are all non-positive sequences, $\left\{a_{n}\right\}_{n \geq 0}$ is said to satisfy the $\ell$-th iterated Turán inequalities [3] or to be $\ell$-log-concave [11. Clearly, 1-log-concavity is just log-concavity.

We focus on the behaviour of a sequence $\left\{a_{n}\right\}_{n \geq 0}$ when $n$ is sufficiently large. Similar to the asymptotic $\ell$-log-concavity given in [8], we say a sequence satisfies the higher order Turán inequalities asymptotically if (1.1) holds for $n$ sufficiently large. We aim to give a criterion on the asymptotic higher order Turán inequalities for $P$-recursive sequences. A $P$-recursive sequence of order $d$ satisfies a recurrence relation of the form

$$
a_{n}=r_{1}(n) a_{n-1}+r_{2}(n) a_{n-1}+\cdots+r_{d}(n) a_{n-d},
$$

where $r_{i}(n)$ are rational functions of $n$, see [14, Section 6.4].

By the asymptotic estimation given by Birkhoff and Trjitzinsky [1] and developed by Wimp and Zeilberger [18, we see that (Theorem 4.1) many $P$-recursive sequences $\left\{a_{n}\right\}_{n \geq 0}$ satisfy

$$
u_{n}=\frac{a_{n-1} a_{n+1}}{a_{n}^{2}}=1+\sum_{i=1}^{m} \frac{r_{i}(\log n)}{n^{\alpha_{i}}}+o\left(\frac{1}{n^{\beta}}\right),
$$

where $m$ is a nonnegative integer, $\alpha_{i}$ are real numbers, $r_{i}(x)$ are rational functions of $x$ and

$$
0<\alpha_{1}<\alpha_{2}<\cdots<\alpha_{m}<\beta .
$$

With the asymptotic form (1.3), we are able to give a sufficient condition on the asymptotic higher order Turán inequalities in Section 2 . Then in Section 3, we extend the result of Hou and Zhang [8] on asymptotic $\ell$-logconcavity. In particular, we apply the criterions on $P$-recursive sequences in Section 4. At last, we give a method to find the $N$ such that for any $n>N$ the higher order Turán inequalities hold in section 5. 


\section{The asymptotic higher order Turán inequalities}

In this section, we will give a sufficient condition on the asymptotic higher order Turán inequalities. $\log n$.

We firstly give an estimation on the difference of rational functions of

Lemma 2.1. Let $r(x)$ be a rational function of $x$ and $K$ be a positive integer.

Then

$$
r(\log (n+1))-r(\log n)=\sum_{i=1}^{K} \frac{r_{i}(\log n)}{n^{i}}+o\left(\frac{1}{n^{K}}\right),
$$

for some rational functions $r_{i}(x)$. Similarly,

$$
r(\log (n-1))-r(\log n)=\sum_{i=1}^{K} \frac{\tilde{r}_{i}(\log n)}{n^{i}}+o\left(\frac{1}{n^{K}}\right),
$$

for some rational functions $\tilde{r}_{i}(x)$. If $r(x)$ is a polynomial, are the $r_{i}(x), \tilde{r}_{i}(x)$ 's. Moreover,

$$
r_{1}(x)=-\tilde{r}_{1}(x)=r^{\prime}(x) \quad \text { and } \quad r_{2}(x)=\tilde{r}_{2}(x)=\frac{r^{\prime \prime}(x)-r^{\prime}(x)}{2} .
$$

Proof. Let

$$
\delta=\log (n+1)-\log n=\sum_{k=1}^{K}(-1)^{k-1} \frac{1}{k n^{k}}+o\left(\frac{1}{n^{K}}\right) .
$$

Notice that $\delta$ behaves like $1 / n$ and for any rational function $f(x)$ we have

$$
\lim _{x \rightarrow \infty} \frac{f(\log x)}{x}=0 .
$$

Therefore,

$$
\begin{aligned}
r(\log (n+1)) & =r((\log n)+\delta) \\
& =\sum_{k=0}^{K} \frac{r^{(k)}(\log n)}{k !} \delta^{k}+o\left(\delta^{K}\right),
\end{aligned}
$$

where $r^{(k)}(x)$ is the $k$-th derivative of $r(x)$. Expanding $\delta^{k}$ by (2.4) and collecting the coefficients of $1 / n^{k}$, we derive that

$$
r(\log (n+1))=r(\log n)+\sum_{k=1}^{K} \frac{r_{k}(\log n)}{n^{k}}+o\left(\frac{1}{n^{K}}\right),
$$

where $r_{k}(x)$ are certain rational functions of $x$. In particular, we have

$$
r_{1}(x)=r^{\prime}(x) \quad \text { and } \quad r_{2}(x)=\frac{r^{\prime \prime}(x)-r^{\prime}(x)}{2},
$$

completing the proof.

Now we are ready to give the main result. 
Theorem 2.2. Let $\left\{a_{n}\right\}_{n \geq 0}$ be a sequence such that (1.3) holds. Assume that $\alpha_{m}-\alpha_{1} \geq 1$. If

$$
0<\alpha_{1}<2 \text { and } r_{1}(x)<0 \text { for } x \text { sufficiently large, }
$$

or

$$
\alpha_{1}=2 \text { and } r_{1}(x)<-1 \text { for } x \text { sufficiently large, }
$$

then $\left\{a_{n}\right\}_{n \geq 0}$ satisfies higher order Turán inequalities asymptotically.

Proof. Dividing $a_{n}^{2} a_{n+1}^{2}$ on both sides of (1.1), we see that the higher order Turán inequality is equivalent to

$$
4\left(1-u_{n}\right)\left(1-u_{n+1}\right)-\left(1-u_{n} u_{n+1}\right)^{2} \geq 0 .
$$

Denote

$$
\alpha=\alpha_{1}, \quad u_{n}=1+\frac{\xi(n)}{n^{\alpha}}
$$

and

$$
f(n)=4\left(1-u_{n}\right)\left(1-u_{n+1}\right)-\left(1-u_{n} u_{n+1}\right)^{2} .
$$

We have

$f(n)=-\frac{t^{2}(n)+\xi(n) \xi(n+1)\left(2 \xi(n+1) n^{\alpha}+2 \xi(n)(n+1)^{\alpha}+\xi(n) \xi(n+1)\right)}{n^{2 \alpha}(n+1)^{2 \alpha}}$,

where

$$
t(n)=\xi(n)(n+1)^{\alpha}-\xi(n+1) n^{\alpha} .
$$

Noting that

$$
\xi(n)=\sum_{i=1}^{m} \frac{r_{i}(\log n)}{n^{\alpha_{i}-\alpha}}+o\left(\frac{1}{n^{\beta-\alpha}}\right)
$$

we have

$$
t(n)=\sum_{i=1}^{m}\left(\frac{(n+1)^{\alpha} r_{i}(\log n)}{n^{\alpha_{i}-\alpha}}-\frac{n^{\alpha} r_{i}(\log (n+1))}{(n+1)^{\alpha_{i}-\alpha}}\right)+o\left(\frac{1}{n^{\beta-2 \alpha}}\right) .
$$

From Lemma 2.1, we derive that

$$
\begin{aligned}
& \frac{(n+1)^{\alpha} r(\log n)}{n^{\gamma}}-\frac{n^{\alpha} r(\log (n+1))}{(n+1)^{\gamma}} \\
= & n^{\alpha-\gamma} r(\log n)\left(\left(1+\frac{1}{n}\right)^{\alpha}-\left(1+\frac{1}{n}\right)^{-\gamma}\left(1+\frac{r(\log (n+1))-r(\log n)}{r(\log n)}\right)\right) \\
= & n^{\alpha-\gamma} r(\log n)\left(\frac{\alpha+\gamma}{n}-\frac{r^{\prime}(\log n)}{n r(\log n)}+o\left(\frac{1}{n}\right)\right) \\
= & n^{\alpha-\gamma-1} r(\log n)(\alpha+\gamma+o(1)),
\end{aligned}
$$


where the last equality holds since $r^{\prime}(x) / r(x) \rightarrow 0$ when $x \rightarrow \infty$ for any rational function $r(x)$. Therefore,

$$
\begin{aligned}
t(n) & =\sum_{i=1}^{m} n^{2 \alpha-\alpha_{i}-1} r_{i}(\log n)\left(\alpha_{i}+o(1)\right)+o\left(\frac{1}{n^{\beta-2 \alpha}}\right) \\
& =\alpha n^{\alpha-1} r_{1}(\log n)(1+o(1)),
\end{aligned}
$$

since $\beta>\alpha_{m} \geq \alpha+1$. Hence,

$$
t^{2}(n)=\alpha^{2} n^{2 \alpha-2}\left(r_{1}(\log n)\right)^{2}(1+o(1)) .
$$

On the other hand, we have

$$
\begin{aligned}
\xi(n) \xi(n+1)\left(2 \xi(n+1) n^{\alpha}+2 \xi(n)(n+1)^{\alpha}\right. & +\xi(n) \xi(n+1)) \\
& =4\left(r_{1}(\log n)\right)^{3} n^{\alpha}(1+o(1)) .
\end{aligned}
$$

If $\alpha<2$, we have $2 \alpha-2<\alpha$ and thus

$$
f(n)=-4\left(\frac{r_{1}(\log n)}{n^{\alpha}}\right)^{3}(1+o(1)),
$$

which is positive for $n$ sufficiently large if $r_{1}(x)<0$ for $x$ sufficiently large.

If $\alpha=2$, we have

$$
f(n)=-4\left(\frac{r_{1}(\log n)}{n^{3}}\right)^{2}\left(r_{1}(\log n)+1+o(1)\right),
$$

which is positive for $n$ sufficiently large if $r_{1}(x)<-1$ for $x$ sufficiently large.

Remark. Let $r(x) \in \mathbb{R}[x]$ be a rational function of $x$ with real coefficients. Suppose $r(x)=p(x) / q(x)$, where $p(x), q(x)$ are polynomials in $x$. Then $r(x)<0$ for $x$ sufficiently large if and only if

$$
\text { lc } p / \text { lc } q<0,
$$

where lc $p$ and lc $q$ denotes the leading coefficients of $p$ and $q$, respectively.

Example 2.1. By Theorem 2.2, when $u_{n}$ are of the following form, the corresponding $\left\{a_{n}\right\}_{n \geq 0}$ satisfies higher order Turán inequalities for $n$ sufficiently large.

$$
1-\frac{1}{n}, \quad 1-\frac{1}{n \log n}, \quad 1-\frac{2}{n^{2}}, \quad 1-\frac{\log n}{n^{2}}
$$

Noting that for $a_{n}=1 / n$ !, we have

$$
u_{n}=\frac{n}{n+1}=1-\frac{1}{n}+\frac{1}{n^{2}}+o\left(n^{-2-\delta}\right), \quad 0<\delta<1,
$$

and hence $\left\{a_{n}\right\}_{n \geq 0}$ satisfies higher order Turán inequalities for $n$ sufficiently large. In fact,

$$
4\left(1-u_{n}\right)\left(1-u_{n+1}\right)-\left(1-u_{n} u_{n+1}\right)^{2}=\frac{4}{(n+1)(n+2)^{2}}>0, \quad \forall n \geq 0 .
$$


Example 2.2. We remark that the condition $\alpha_{m} \geq \alpha_{1}+1$ is necessary.

Let $a_{0}=a_{1}=1$ and $a_{n+1}=a_{n}^{2} u_{n} / a_{n-1}$ for $n \geq 1$ where

$$
u_{n}= \begin{cases}1-\frac{1}{n}, & n \text { is even, } \\ 1-\frac{1}{n}+\frac{1}{n^{4 / 3}}, & n \text { is odd. }\end{cases}
$$

We have

$$
a_{2}=1, a_{3}=\frac{1}{2}, a_{4}=\frac{1}{36}\left(6+3^{2 / 3}\right), a_{5}=\frac{1}{864}\left(6+3^{2 / 3}\right)^{2}, \ldots
$$

Clearly,

$$
u_{n}=1-\frac{1}{n}+o\left(\frac{1}{n}\right)
$$

However,

$$
4\left(1-u_{n}\right)\left(1-u_{n+1}\right)-\left(1-u_{n} u_{n+1}\right)^{2}=-\frac{1}{n^{8 / 3}}(1+o(1)),
$$

and hence $\left\{a_{n}\right\}_{n \geq 0}$ does not satisfy the higher order Turán inequalities when $n$ sufficiently large.

Example 2.3. Let $a_{n}=n \log n$, we see that

$$
u_{n}=1+\frac{-1-\frac{1}{\log n}-\frac{1}{(\log n)^{2}}}{n^{2}}+o\left(\frac{1}{n^{3+\delta}}\right),
$$

for any $0<\delta<1$. Hence $\left\{a_{n}\right\}_{n \geq 1}$ satisfies the higher order Turán inequalities asymptotically.

\section{Asymptotic $\ell$-log-concavity}

Hou and Zhang [8] gave a criterion on the $\ell$-log-concavity of sequences $\left\{a_{n}\right\}_{n \geq 0}$ such that the $r_{i}(x)$ 's in (1.3) are all constants. In this section, we will give a similar criterion for the general case where the $r_{i}(x)$ 's are arbitrary rational functions of $x$.

We first give an estimate on the ratio $a_{n-1} a_{n+1} / a_{n}^{2}$ for a special kind of $a_{n}$.

Lemma 3.1. Let

$$
a_{n}=\frac{r(\log n)}{n^{\alpha}},
$$

where $r(x)$ is a rational function of $x$ and $\alpha$ be a positive real number. Then for any integer $K \geq 2$, there exist rational functions $r_{i}(x)$ of $x$ such that

$$
u_{n}=\frac{a_{n-1} a_{n+1}}{a_{n}^{2}}=1+\sum_{i=2}^{K} \frac{r_{i}(\log n)}{n^{i}}+o\left(\frac{1}{n^{K}}\right) .
$$


At the same time, there exist rational functions $\tilde{r}_{i}(x)$ of $x$ such that

$$
a_{n+1}+a_{n-1}-2 a_{n}=\sum_{i=2}^{K} \frac{\tilde{r}_{i}(\log n)}{n^{i+\alpha}}+o\left(\frac{1}{n^{K+\alpha}}\right) .
$$

Moreover, we have

$$
r_{2}(x)=\alpha+(\log r(x))^{\prime \prime}-(\log r(x))^{\prime}
$$

and

$$
\tilde{r}_{2}(x)=\alpha(\alpha+1) r(x)-(2 \alpha+1) r^{\prime}(x)+r^{\prime \prime}(x) .
$$

Proof. Denote $\log n, \log (n+1)$, and $\log (n-1)$ by $l, l^{+}$and $l^{-}$, respectively. We have

$$
\begin{aligned}
u_{n} & =\frac{n^{2 \alpha}}{(n-1)^{\alpha}(n+1)^{\alpha}} \frac{r\left(l^{+}\right) r\left(l^{-}\right)}{r^{2}(l)} \\
& =\left(1-\frac{1}{n^{2}}\right)^{-\alpha}\left(1+\frac{r\left(l^{+}\right)-r(l)}{r(l)}\right)\left(1+\frac{r\left(l^{-}\right)-r(l)}{r(l)}\right) .
\end{aligned}
$$

By Lemma 2.1, we derive that

$$
\begin{aligned}
u_{n}= & \left(1+\sum_{i=1}^{K}\left(\begin{array}{c}
-\alpha \\
i
\end{array}\right)\left(-\frac{1}{n^{2}}\right)^{i}+o\left(\frac{1}{n^{K}}\right)\right)\left(1+\frac{1}{r(l)} \sum_{i=1}^{K} \frac{p_{i}(l)}{n^{i}}+o\left(\frac{1}{n^{K}}\right)\right) \\
& \left(1+\frac{1}{r(l)} \sum_{i=1}^{K} \frac{q_{i}(l)}{n^{i}}+o\left(\frac{1}{n^{K}}\right)\right) \\
= & 1+\frac{\alpha}{n^{2}}+\frac{r(l)\left(r^{\prime \prime}(l)-r^{\prime}(l)\right)-\left(r^{\prime}(l)\right)^{2}}{n^{2} r^{2}(l)}+\cdots+o\left(\frac{1}{n^{K}}\right),
\end{aligned}
$$

where $p_{i}(x), q_{i}(x)$ are rational functions of $x$.

At the same time, we have

$$
\begin{gathered}
a_{n+1}+a_{n-1}-2 a_{n} \\
=\frac{r(l)}{n^{\alpha}}\left(\left(1+\frac{1}{n}\right)^{-\alpha}\left(1+\frac{r\left(l^{+}\right)-r(l)}{r(l)}\right)\right. \\
\left.\quad+\left(1-\frac{1}{n}\right)^{-\alpha}\left(1+\frac{r\left(l^{-}\right)-r(l)}{r(l)}\right)-2\right) \\
=\frac{r(l)}{n^{\alpha}}\left(\sum_{i=0}^{K} \frac{\left(\begin{array}{c}
-\alpha \\
i
\end{array}\right)}{n^{i}}\left(1+\frac{1}{r(l)} \sum_{i=1}^{K} \frac{p_{i}(l)}{n^{i}}\right)\right. \\
\left.\quad+\sum_{i=0}^{K} \frac{\left(\begin{array}{c}
-\alpha \\
i
\end{array}\right)}{(-n)^{i}}\left(1+\frac{1}{r(l)} \sum_{i=1}^{K} \frac{q_{i}(l)}{n^{i}}\right)-2+o\left(\frac{1}{n^{K}}\right)\right) \\
=\frac{r(l)}{n^{\alpha}}\left(\frac{\alpha(\alpha+1)}{n^{2}}-\frac{2 \alpha r^{\prime}(l)}{n^{2} r(l)}+\frac{r^{\prime \prime}(l)-r^{\prime}(l)}{n^{2} r(l)}+\cdots+o\left(\frac{1}{n^{K}}\right)\right),
\end{gathered}
$$


completing the proof.

Now we are ready to give a criterion on the asymptotic $\ell$-log-concavity.

Theorem 3.2. Let $\left\{a_{n}\right\}_{n \geq 0}$ be a sequences such that (1.3) holds and denote $\ell=\left\lfloor\alpha_{m} / \alpha_{1}\right\rfloor$. If

$$
0<\alpha_{1}<2 \text { and } r_{1}(x)<0 \text { for } x \text { sufficiently large, }
$$

or

$$
\alpha_{1}=2 \text { and } r_{1}(x)<-2+1 / 2^{r-2} \text { for } x \text { sufficiently large, }
$$

then $\left\{a_{n}\right\}_{n \geq 0}$ is asymptotically $\ell$-log-concave.

Proof. Let

$$
u_{n}=\frac{a_{n-1} a_{n+1}}{a_{n}^{2}}, \quad b_{n}=a_{n}^{2}-a_{n-1} a_{n+1} .
$$

It is easy to check that

$$
\frac{b_{n-1} b_{n+1}}{b_{n}^{2}}=u_{n}^{2} \frac{\left(u_{n-1}-1\right)\left(u_{n+1}-1\right)}{\left(u_{n}-1\right)^{2}} .
$$

We will give an estimation for this ratio.

Denote $\alpha=\alpha_{1}$ and $r(x)=r_{1}(x)$. Since $u_{n}$ is of form (1.3), so is $u_{n}^{2}$. Moreover,

$$
u_{n}^{2}=1+\frac{2 r(\log n)}{n^{\alpha}}+\cdots+o\left(\frac{1}{n^{\beta}}\right) .
$$

Rewrite $u_{n}$ as $u_{n}=1+f_{n} g_{n}$ with

$$
f_{n}=\frac{r(x)}{n^{\alpha}} \quad \text { and } \quad g_{n}=1+\sum_{i=2}^{m} \frac{r_{i}(x)}{r(x) n^{\alpha_{i}-\alpha}}+o\left(\frac{1}{n^{\beta-\alpha}}\right) .
$$

Then we have

$$
\frac{\left(u_{n-1}-1\right)\left(u_{n+1}-1\right)}{\left(u_{n}-1\right)^{2}}=\frac{f_{n-1} f_{n+1}}{f_{n}^{2}} \frac{g_{n-1} g_{n+1}}{g_{n}^{2}} .
$$

By Lemma 3.1, we get that

$$
\frac{f_{n-1} f_{n+1}}{f_{n}^{2}}=1+\frac{t(\log n)}{n^{2}}+\cdots+o\left(\frac{1}{n^{\beta}}\right),
$$

with

$$
t(x)=\alpha+(\log r(x))^{\prime \prime}-(\log r(x))^{\prime} .
$$

To estimate the ratio $g_{n-1} g_{n+1} / g_{n}^{2}$, we consider $h_{n}=\log g_{n}$. By the Taylor expansion of $\log (1+x)$, we derive that $h_{n}$ is of form

$$
\sum_{i=1}^{l} \frac{s_{i}(\log n)}{n^{\beta_{i}}}+o\left(\frac{1}{n^{\beta-\alpha}}\right)
$$


where

$$
\alpha_{2}-\alpha=\beta_{1}<\beta_{2}<\ldots<\beta_{l}<\beta-\alpha,
$$

and $s_{i}(x)$ are rational functions of $x$. Moreover, $s_{1}(x)=r_{2}(x) / r(x)$. By Lemma 3.1, we see that

$$
\begin{aligned}
\log \frac{g_{n+1} g_{n-1}}{g_{n}^{2}} & =h_{n+1}+h_{n-1}-2 h_{n} \\
& =\sum_{i=1}^{l} \sum_{j=2}^{K} \frac{s_{i j}(\log n)}{n^{j+\beta_{i}}}+o\left(\frac{1}{n^{\beta-\alpha}}\right),
\end{aligned}
$$

where $K$ is an integer with $K>\beta-\alpha$ and $s_{i j}(x)$ are rational functions of $x$. By the Taylor expansion of $e^{x}$, we see that

$$
\frac{g_{n+1} g_{n-1}}{g_{n}^{2}}=1+\sum_{i=1}^{d} \frac{\tilde{s}_{i}(\log n)}{n^{\gamma_{i}}}+o\left(\frac{1}{n^{\beta-\alpha}}\right),
$$

where

$$
\alpha_{2}-\alpha+2=\gamma_{1}<\gamma_{2}<\ldots<\gamma_{d}<\beta-\alpha,
$$

and $\tilde{s}_{i}(x)$ are rational functions of $x$.

Combining (3.7), (3.8) and (3.9) together, we derive that $b_{n+1} b_{n-1} / b_{n}^{2}$ is of form (1.3) and

$$
\begin{aligned}
\frac{b_{n+1} b_{n-1}}{b_{n}^{2}}=\left(1+\frac{2 r(\log n)}{n^{\alpha}}\right. & \left.+\cdots+o\left(\frac{1}{n^{\beta}}\right)\right) \\
& \times\left(1+\frac{t(\log n)}{n^{2}}+\cdots+o\left(\frac{1}{n^{\beta-\alpha}}\right)\right) .
\end{aligned}
$$

When $\alpha<2$, we have

$$
\frac{b_{n+1} b_{n-1}}{b_{n}^{2}}=1+\frac{2 r(\log n)}{n^{\alpha}}+\cdots+o\left(\frac{1}{n^{\beta-\alpha}}\right) .
$$

If $r(x)<0$ for $x$ sufficiently large, so is $2 r(x)$. If $\alpha_{m} \geq 2 \alpha$, we have $\beta-\alpha>\alpha$. Hence the ratio $b_{n-1} b_{n+1} / b_{n}^{2}$ is less than 1 for $n$ sufficiently large, implying that $\left\{a_{n}\right\}_{n \geq 0}$ is asymptotically 2-log-concave. Repeating this argument, we finally derive that $\left\{a_{n}\right\}_{n \geq 0}$ is $\ell$-log-concave.

Then we consider the case of $\alpha=2$. It is clear that if $r(x)<0$ for $x$ sufficiently large, then $\left\{a_{n}\right\}_{n \geq 0}$ is log-concave. Now suppose that $r(x)<-1$ for $x$ sufficiently large and $\alpha_{m} \geq 4$. We have

$$
\frac{b_{n+1} b_{n-1}}{b_{n}^{2}}=1+\frac{2 r(\log n)+t(\log n)}{n^{2}}+\cdots+o\left(\frac{1}{n^{\beta-2}}\right) .
$$

If

$$
\lim _{x \rightarrow \infty} r(x)=a<-1
$$


we have

$$
\lim _{x \rightarrow \infty} 2 r(x)+t(x)=2 a+2<0,
$$

since

$$
\lim _{x \rightarrow \infty}(\log r(x))^{\prime}=\lim _{x \rightarrow \infty} \frac{r^{\prime}(x)}{r(x)}=0,
$$

and

$$
\lim _{x \rightarrow \infty}(\log r(x))^{\prime \prime}=\lim _{x \rightarrow \infty} \frac{\left(r^{\prime}(x) / r(x)\right)^{\prime}}{r^{\prime}(x) / r(x)} \lim _{x \rightarrow \infty} \frac{r^{\prime}(x)}{r(x)}=0
$$

If

$$
\lim _{x \rightarrow \infty} r(x)=-1
$$

we have

$$
\begin{aligned}
\lim _{x \rightarrow \infty} \frac{(\log r(x))^{\prime \prime}-(\log r(x))^{\prime}}{2 r(x)+2} & =\lim _{x \rightarrow \infty} \frac{r^{\prime}(x) / r(x)}{2 r(x)+2} \lim _{x \rightarrow \infty} \frac{(\log r(x))^{\prime \prime}-(\log r(x))^{\prime}}{r^{\prime}(x) / r(x)} \\
& =\lim _{x \rightarrow \infty} \frac{\left(r^{\prime}(x) / r(x)\right)^{\prime}}{2 r^{\prime}(x)} \cdot(-1)=0 .
\end{aligned}
$$

Hence

$$
2 r(x)+t(x)=(2 r(x)+2)(1+o(1))<0
$$

for $x$ sufficiently large, implying that $\left\{a_{n}\right\}_{n \geq 0}$ is 2-log-concave for $n$ sufficiently large.

In general, if $r(x)<-2+1 / 2^{\ell-2}$ for $x$ sufficiently large, we have

$$
2 r(x)+t(x)<-2+\frac{1}{2^{\ell-3}}
$$

for $n$ sufficiently large. Repeating this discussion, we finally derive that $\left\{a_{n}\right\}_{n \geq 0}$ is asymptotically $\ell$-log-concave.

We see that the condition for the higher order Turán inequalities given by Theorem 2.2 and the condition for the 2-log-concavity given by Theorem 3.2 coincide.

Example 3.1. Let $a_{n}=n^{\alpha}(\alpha \geq 2)$, we see that

$$
u_{n}=\left(1-\frac{1}{n^{2}}\right)^{\alpha}=1-\frac{\alpha}{n^{2}}+\sum_{i=2}^{\infty} \frac{\left(\begin{array}{c}
\alpha \\
i
\end{array}\right)(-1)^{i}}{n^{2 i}} .
$$

Hence $\left\{a_{n}\right\}_{n \geq 0}$ is asymptotically $\ell$-log-concave for any positive integer $\ell$.

Example 3.2. Let $a_{n}=n^{2} \log n$, we see that

$$
u_{n}=1+\frac{-2-\frac{1}{\log n}-\frac{1}{(\log n)^{2}}}{n^{2}}+\sum_{i=2}^{\infty} \frac{r_{i}(\log n)}{n^{2 i}},
$$

where $r_{i}(x)$ are rational functions of $x$. Hence $\left\{a_{n}\right\}_{n \geq 1}$ is asymptotically $\ell$-log-concave for any positive integer $\ell$. 


\section{$4 \quad P$-recursive sequences and asymptotic higher or- der Turán property}

In this section, we apply the criterion given in the previous section to $P$ recursive sequences. Recall that a $P$-recursive sequence of order $d$ satisfies a recurrence relation of the form

$$
a_{n}=r_{1}(n) a_{n-1}+r_{2}(n) a_{n-1}+\cdots+r_{d}(n) a_{n-d},
$$

where $r_{i}(n)$ are rational functions of $n$, see [14, Section 6.4]. We will use the asymptotic estimation of $P$-recursive sequences given by Birkhoff and Trjitzinsky [1] and developed by Wimp and Zeilberger [18]. They showed that a $P$-recursive sequence is asymptotically equal to a linear combination of terms of the form

$$
e^{Q(\rho, n)} s(\rho, n)
$$

where

$$
Q(\rho, n)=\mu_{0} n \log n+\sum_{j=1}^{\rho} \mu_{j} n^{j / \rho},
$$

and

$$
s(\rho, n)=n^{r} \sum_{j=0}^{t-1}(\log n)^{j} \sum_{s=0}^{M-1} b_{s j} n^{-s / \rho},
$$

with $\rho, t, M$ being positive integers and $\mu_{j}, r, b_{s j}$ being complex numbers.

The following theorem shows that the $u_{n}$ of a $P$-recursive sequence is of form (1.3).

Theorem 4.1. Let $\left\{a_{n}\right\}_{n \geq 0}$ be a P-recursive sequence with asymptotic form (4.11). Then $u_{n}=a_{n-1} a_{n+1} / a_{n}^{2}$ is of the form (1.3) when $n$ tends to infinity.

Proof. Noting that the product of two terms of the form (1.3) is still of the form (1.3), we can treat each factor of (4.11) separately. The factors $Q(\rho, n)$ and $n^{r}$ has been discussed in [8, Theorem 3.1]. We need only consider the term

$$
f_{n}=\sum_{s=0}^{M} \sum_{j=0}^{t-1} b_{s j} \frac{(\log n)^{j}}{n^{s / \rho}}+o\left(\frac{1}{n^{\beta}}\right),
$$

where $b_{0 j}$ are not all zeros and $\beta$ is a real number satisfying

$$
(M+1) / \rho>\beta>M / \rho .
$$

Rewrite $f_{n}$ as

$$
f_{n}=\sum_{s=0}^{M} \frac{p_{s}(\log n)}{n^{s / \rho}}+o\left(\frac{1}{n^{\beta}}\right)
$$


where

$$
p_{s}(x)=\sum_{j=0}^{t-1} b_{s j} x^{j}
$$

are polynomials in $x$. By Lemma 2.1, we have

$$
\begin{aligned}
f_{n+1} & =\sum_{s=0}^{M} \frac{p_{s}(\log (n+1))}{(n+1)^{s / \rho}}+o\left(\frac{1}{n^{\beta}}\right) \\
& =\sum_{s=0}^{M} \frac{p_{s}(\log n)+\sum_{j=1}^{M} \frac{q_{s j}(\log n)}{n^{j}}+o\left(\frac{1}{n^{M}}\right)}{n^{s / \rho}}\left(1+\frac{1}{n}\right)^{-s / \rho}+o\left(\frac{1}{n^{\beta}}\right) \\
& =f_{n}+\sum_{s=0}^{M} \frac{p_{s}^{\prime}(\log n)-s p_{s}(\log n) / \rho}{n^{1+s / \rho}}+\sum_{s=0}^{M} \frac{r_{s}(\log n)}{n^{2+s / \rho}}+o\left(\frac{1}{n^{\beta}}\right),
\end{aligned}
$$

where $q_{s j}(x), r_{s}(x)$ are polynomials in $x$. Similarly, we have

$$
\begin{aligned}
f_{n-1} & =\sum_{s=0}^{M} \frac{p_{s}(\log (n-1))}{(n-1)^{s / \rho}}+o\left(\frac{1}{n^{\beta}}\right) \\
& =\sum_{s=0}^{M} \frac{p_{s}(\log n)+\sum_{j=1}^{M} \frac{\tilde{q}_{s j}(\log n)}{n^{j}}+o\left(\frac{1}{n^{M}}\right)}{n^{s / \rho}}\left(1-\frac{1}{n}\right)^{-s / \rho}+o\left(\frac{1}{n^{\beta}}\right) \\
& =f_{n}-\sum_{s=0}^{M} \frac{p_{s}^{\prime}(\log n)-s p_{s}(\log n) / \rho}{n^{1+s / \rho}}+\sum_{s=0}^{M} \frac{\tilde{r}_{s}(\log n)}{n^{2+s / \rho}}+o\left(\frac{1}{n^{\beta}}\right)
\end{aligned}
$$

where $\tilde{q}_{s j}(x), \tilde{r}_{s}(x)$ are polynomials in $x$. Since $p_{0}(x) \neq 0$, we have

$$
\frac{1}{f_{n}^{2}} \cdot o\left(\frac{1}{n^{\beta}}\right)=o\left(\frac{1}{n^{\beta^{\prime}}}\right)
$$

for any $\beta^{\prime}<\beta$. Therefore,

$$
\begin{aligned}
\frac{f_{n+1} f_{n-1}}{f_{n}^{2}}=1 & -\frac{1}{f_{n}^{2}}\left(\sum_{s=0}^{M} \frac{p_{s}^{\prime}(\log n)-s p_{s}(\log n) / \rho}{n^{1+s / \rho}}\right)^{2}+\frac{1}{f_{n}^{2}} \sum_{s=0}^{M} \frac{t_{s}(\log n)}{n^{2+s / \rho}} \\
& +o\left(\frac{1}{n^{\beta^{\prime}}}\right) \\
=1 & +\sum_{s=0}^{M} \frac{\tilde{t}_{s}(\log n)}{n^{2+s / \rho}}+o\left(\frac{1}{n^{\beta^{\prime}}}\right)
\end{aligned}
$$

where $t_{s}(x)$ are polynomials in $x$ and $\tilde{t}_{s}(x)$ are rational functions of $x$.

We give some examples.

Example 4.1. Let

$$
C_{n}=\frac{1}{n+1}\left(\begin{array}{c}
2 n \\
n
\end{array}\right)
$$


be the Catalan numbers. Its inverse $a_{n}=1 / C_{n}$ satisfies

$$
(4 n+2) a_{n+1}-(n+2) a_{n}=0 .
$$

By packages such as Asyrec given by Zeilberger [19], asymptotics.m given by Kauers [9], or P-rec.m given by Hou and Zhang [8], we find that

$$
a_{n}=c \cdot \frac{n^{3 / 2}}{4^{n}}\left(1+\frac{9}{8 n}+\frac{17}{128 n^{2}}+o\left(\frac{1}{n^{2}}\right)\right),
$$

where $c$ is a constant. (In fact, $c=\sqrt{\pi}$.) Therefore,

$$
u_{n}=1-\frac{3}{2 n^{2}}+\frac{9}{4 n^{3}}-\frac{21}{8 n^{4}}+o\left(\frac{1}{n^{4}}\right) .
$$

Hence $\left\{1 / C_{n}\right\}_{n \geq 0}$ satisfies the higher order Turán inequalities asymptotically and is asymptotically 2-log-concave.

Example 4.2. Let $I_{n}$ be the number of involutions on $\{1, \ldots, n\}$, i.e., permutations $\sigma$ such that $\sigma^{2}=\mathrm{id}$. It is well-known that

$$
I_{n}=I_{n-1}+(n-1) I_{n-2} .
$$

Hence, $a_{n}=I_{n} / n$ ! satisfies the recurrence relation

$$
n a_{n}=a_{n-1}+a_{n-2} .
$$

By the above packages, we find that

$$
a_{n}=c \cdot \frac{e^{n / 2+\sqrt{n}}}{n^{n / 2}} \frac{1}{\sqrt{n}}\left(1+\frac{7}{24 \sqrt{n}}-\frac{215}{1152 n}+o\left(\frac{1}{n}\right)\right) .
$$

Therefore,

$$
u_{n}=1-\frac{1}{2 n}-\frac{1}{4 n^{3 / 2}}+\frac{5}{8 n^{2}}+o\left(\frac{1}{n^{2}}\right),
$$

and thus $\left\{I_{n} / n !\right\}_{n \geq 0}$ satisfies the higher order Turán inequalities asymptotically and is asymptotically $\ell$-log-concave for any positive integer $\ell$.

Let $A_{n}$ be the $n$-th Apéry number given by

$$
A_{n}=\sum_{k=0}^{n}\left(\begin{array}{l}
n \\
k
\end{array}\right)^{2}\left(\begin{array}{c}
n+k \\
k
\end{array}\right)^{2} .
$$

By a similar discussion, we derive that $A_{n} / n$ ! satisfies the higher order Turán inequalities asymptotically and is asymptotically $\ell$-log-concave for any positive integer $\ell$. 


\section{The higher order Turán inequality}

In this section, we will give a method to find the explicit $N$ such that the higher order Turán inequalities holds for $\left\{a_{n}\right\}_{n \geq N}$ when $\left\{a_{n}\right\}_{n \geq 0}$ is $P$ recursive.

The following lemma indicates that to prove the higher order Turán inequality, we need only consider the boundary points.

Lemma 5.1. Let

$$
t(x, y)=4(1-x)(1-y)-(1-x y)^{2} .
$$

Suppose that there exist $x_{1}<x_{2}$ and $y_{1}<y_{2}$ such that

$$
t\left(x_{1}, y_{1}\right)>0, \quad t\left(x_{1}, y_{2}\right)>0, \quad t\left(x_{2}, y_{1}\right)>0, \quad t\left(x_{2}, y_{2}\right)>0 .
$$

Then for every $x$ and $y$ such that

$$
x_{1}<x<x_{2}, \quad y_{1}<y<y_{2},
$$

we have $t(x, y)>0$.

Proof. It's easy to see

$$
t\left(x, y_{1}\right)=-y_{1}^{2} x^{2}+\left(6 y_{1}-4\right) x+3-4 y_{1}
$$

Since $-y_{1}^{2} \leq 0$ and $t\left(x_{1}, y_{1}\right)>0, t\left(x_{2}, y_{1}\right)>0$, for any $x \in\left(x_{1}, x_{2}\right)$, we have $t\left(x, y_{1}\right)>0$. Similarly, $t\left(x, y_{2}\right)>0$ for any $x \in\left(x_{1}, x_{2}\right)$.

We also can rewrite $t(x, y)$ as

$$
t(x, y)=-x^{2} y^{2}+(6 x-4) y+3-4 x .
$$

Since $-x^{2} \leq 0$ and $t\left(x, y_{1}\right)>0, t\left(x, y_{2}\right)>0$, we have $t(x, y)>0$ for any $y \in\left(y_{1}, y_{2}\right)$.

As a natural consequence, we derive that

Theorem 5.2. Let

$$
t(x, y)=4(1-x)(1-y)-(1-x y)^{2} \quad \text { and } \quad u_{n}=\frac{a_{n-1} a_{n+1}}{a_{n}^{2}} .
$$

If there exist an integer $N$, an upper bound $f_{n}$ and a lower bound $g_{n}$ of $u_{n}$ such that for all $n \geq N$,

$$
g_{n}<u_{n}<f_{n},
$$

and

$$
t\left(g_{n}, g_{n+1}\right)>0, \quad t\left(g_{n}, f_{n+1}\right)>0, \quad t\left(f_{n}, g_{n+1}\right)>0, \quad t\left(f_{n}, f_{n+1}\right)>0 .
$$

Then $\left\{a_{n}\right\}_{n \geq N}$ satisfies the higher order Turán inequality. 
When $\left\{a_{n}\right\}$ has the asymptotic expression (4.11) such that $t=1$, we are able to compute the bounds $f_{n}$ and $g_{n}$ by the following HT algorithm. We implemented the HT algorithm in the package P-rec.m which is available at [20]. Here we give an outline of the algorithm.

\section{The HT Algorithm}

INPUT: a difference operator $L$ which annihilates $a_{n}$, initial values $a_{0}, a_{1}, \ldots, a_{m}$, an integer $K$.

OUTPUT: An integer $N$, rational functions $g_{n}$ and $f_{n}$ such that

$$
g_{n}<\frac{a_{n-1} a_{n+1}}{a_{n}^{2}}<f_{n}, \quad n>N .
$$

1. Find the asymptotic expansion of $a_{n}$ up to $K$ terms.

2. Denote $r_{n}=\frac{a_{n}}{a_{n-1}}$, compute the asymptotic expression of $r_{n}$

$$
r_{n} \approx \sum_{i=1}^{K} \frac{c_{i}}{n^{\alpha_{i}}}
$$

where $c_{i}, \alpha_{i}$ are real numbers .

3. Use the method in 8 to find $N_{1}$ such that

$$
s_{l}(n)<\frac{a_{n}}{a_{n-1}}<s_{u}(n), \quad n>N_{1} .
$$

where

$$
s_{u}(n)=\sum_{i=1}^{K} \frac{c_{i}}{n^{\alpha_{i}}}+\frac{1}{n^{\alpha_{K}}}, \quad s_{l}(n)=\sum_{i=1}^{K} \frac{c_{i}}{n^{\alpha_{i}}}-\frac{1}{n^{\alpha_{K}}} .
$$

4. Denote $u_{n}=\frac{a_{n+1} a_{n-1}}{a_{n}^{2}}$, compute the asymptotic expression of $u_{n}$

$$
u_{n} \approx 1+\sum_{i=1}^{K-1} \frac{d_{i}}{n^{\beta_{i}}}
$$

where $d_{i}$ are real numbers and $\beta_{i}$ are positive real numbers.

5 . Let

$$
f_{n}=1+\sum_{i=1}^{K-1} \frac{d_{i}}{n^{\beta_{i}}}+\frac{1}{n^{\beta_{K-1}}}, \quad g_{n}=1+\sum_{i=1}^{K-1} \frac{d_{i}}{n^{\beta_{i}}}-\frac{1}{n^{\beta_{K-1}}} .
$$

Find $N_{2}$ such that

$$
\frac{s_{u}(n+1)}{s_{l}(n)}<f_{n}, \quad \frac{s_{l}(n+1)}{s_{u}(n)}>g_{n}, \quad n>N_{2} .
$$

6. Return

$$
\left\{g_{n}, \quad f_{n}, \quad \max \left\{N_{1}, N_{2}\right\}\right\} .
$$


Now I'd like to show the usage of the package.

Example 5.1. Suppose $\left\{a_{n}\right\}$ is defined by

$$
a_{n}=\sum_{k=0}^{n}\left(\begin{array}{l}
n \\
k
\end{array}\right)^{4} .
$$

Then by Zeilberger's algorithm we find that $\left\{a_{n}\right\}$ satisfies the recurrence relation:

$(n+2)^{3} a_{n+2}-2\left(3 n^{2}+9 n+7\right)(2 n+3) a_{n+1}-4(n+1)(4 n+3)(4 n+5) a_{n}=0$,

with the initial values

$$
a_{0}=1, \quad a_{1}=2, \quad a_{2}=18 .
$$

By the command

$H T\left[(n+2)^{3} N^{2}-2\left(3 n^{2}+9 n+7\right)(2 n+3) N-4(n+1)(4 n+3)(4 n+5), n, N,\{1,2,18\}, 4\right]$

We get

$$
\left\{\left\{1+\frac{1}{2 n^{2}}, 1+\frac{5}{2 n^{2}}\right\}, 94\right\}
$$

which means

$$
1+\frac{1}{2 n^{2}}<\frac{a_{n+1} a_{n-1}}{a_{n}^{2}}<1+\frac{1}{2 n^{2}}, \quad n \geq 94
$$

Now we use Theorem 5.2 to prove the higher order Turán inequalities given in [16]. Our method can treat those $P$-recursive sequences of order 2 directly. Moreover, we can treat $P$-recursive sequences of higher order.

Example 5.2. (Theorem 3.2 of [16]) Let $M_{n}$ be the Motzkin numbers given by

$$
(n+4) M_{n+2}-(2 n+5) M_{n+1}-3(n+1) M_{n}=0, \quad n \geq 0,
$$

with the initial values

$$
M_{0}=1, \quad M_{1}=1 .
$$

Then the sequence $\left\{\frac{M_{n}}{n !}\right\}_{n \geq 1}$ satisfies the higher order Turán inequality.

Proof. By the algorithm HT, we derive

$$
1+\frac{1}{2 n^{2}}<\frac{M_{n+1} M_{n-1}}{M_{n}^{2}}<1+\frac{5}{2 n^{2}}, \quad n \geq 75 .
$$

Let

$$
a_{n}=\frac{M_{n}}{n !}, \quad u_{n}=\frac{a_{n+1} a_{n-1}}{a_{n}^{2}} .
$$

Then we have

$$
\frac{n}{n+1}\left(1+\frac{1}{2 n^{2}}\right)<u_{n}<\frac{n}{n+1}\left(1+\frac{5}{2 n^{2}}\right), \quad n \geq 75 .
$$


Let

$$
g_{n}=\frac{n}{n+1}\left(1+\frac{1}{2 n^{2}}\right) \quad \text { and } \quad f_{n}=\frac{n}{n+1}\left(1+\frac{5}{2 n^{2}}\right) .
$$

Direct computation shows that

$$
t\left(g_{n}, g_{n+1}\right)=\frac{64 n^{5}+96 n^{4}+16 n^{3}-24 n^{2}-8 n-9}{16 n^{2}(n+1)^{4}(n+2)^{2}},
$$

which is positive for $n>0$.

Similarly, we have

$$
t\left(g_{n}, f_{n+1}\right)=\frac{64 n^{5}-224 n^{4}-240 n^{3}+24 n^{2}+152 n-49}{16 n^{2}(n+1)^{4}(n+2)^{2}},
$$

which is positive for $n>4$.

$$
t\left(f_{n}, g_{n+1}\right)=\frac{64 n^{5}-96 n^{4}-496 n^{3}-680 n^{2}-520 n-225}{16 n^{2}(n+1)^{4}(n+2)^{2}},
$$

which is positive for $n>4$.

$$
t\left(f_{n}, f_{n+1}\right)=\frac{64 n^{5}-288 n^{4}+272 n^{3}+264 n^{2}-360 n-1225}{16 n^{2}(n+1)^{4}(n+2)^{2}},
$$

which is positive for $n>3$.

Checking the initial values, we finally get that $\left\{\frac{M_{n}}{n !}\right\}_{n \geq 1}$ satisfies the higher order Turán inequality.

Example 5.3. (Theorem 3.12 of [16]) Let $F_{n}^{(3)}$ be the Franel numbers of order 3 given by

$$
(n+2)^{2} F_{n+2}^{(3)}-\left(7 n^{2}+21 n+16\right) F_{n+1}^{(3)}-8(n+1)^{2} F_{n}^{(3)}=0, \quad n \geq 0,
$$

with the initial values

$$
F_{0}^{(3)}=1, \quad F_{1}^{(3)}=2 .
$$

Then the sequence $\left\{\frac{F_{n}^{(3)}}{n !}\right\}_{n \geq 1}$ satisfies the higher order Turán inequality.

Proof. By the algorithm HT, we derive

$$
1<\frac{F_{n+1}^{(3)} F_{n-1}^{(3)}}{\left(F_{n}^{(3)}\right)^{2}}<1+\frac{2}{n^{2}}, \quad n \geq 16 .
$$

Let

$$
a_{n}=\frac{F_{n}^{(3)}}{n !}, \quad u_{n}=\frac{a_{n+1} a_{n-1}}{a_{n}^{2}} .
$$

Then we have

$$
\frac{n}{n+1}<u_{n}<\frac{n}{n+1}\left(1+\frac{2}{n^{2}}\right), \quad n \geq 16
$$


Let

$$
g_{n}=\frac{n}{n+1}, \quad \text { and } \quad f_{n}=\frac{n}{n+1}\left(1+\frac{2}{n^{2}}\right) .
$$

Direct computation shows that

$$
t\left(g_{n}, g_{n+1}\right)=\frac{4}{(n+1)(n+2)^{2}},
$$

which is positive for $n \geq 0$.

Similarly, we have

$$
\begin{gathered}
t\left(g_{n}, f_{n+1}\right)=\frac{4 n^{3}-8 n^{2}-20 n-12}{(n+1)^{4}(n+2)^{2}}>0, \quad n>3 . \\
t\left(f_{n}, g_{n+1}\right)=\frac{4 n^{2}-12 n-4}{n^{2}(n+1)(n+2)^{2}}>0, \quad n>3 . \\
t\left(f_{n}, f_{n+1}\right)=\frac{4 n^{5}-12 n^{4}+4 n^{3}+12 n^{2}-8 n-36}{n^{2}(n+1)^{4}(n+2)^{2}}>0, \quad n>2 .
\end{gathered}
$$

Checking the initial values, we finally get that $\left\{\frac{F_{n}^{(3)}}{n !}\right\}_{n \geq 1}$ satisfies the higher order Turán inequality.

Use the same method, we also can prove that $\left\{\frac{F_{n}}{n !}\right\}_{n \geq 3}$ and $\left\{\frac{D_{n}}{n !}\right\}_{n \geq 0}$ satisfy the higher order Turán inequality, where $F_{n}$ is the Fine number and $D_{n}$ is the Domb number. These properties have been proved in [16].

Example 5.4. Consider the sequences $\left\{b_{n}\right\}_{n \geq 0}$ given by

$$
b_{n}=\sum_{k=0}^{n} \frac{\left(\begin{array}{c}
n \\
k
\end{array}\right)\left(\begin{array}{c}
n+k \\
k
\end{array}\right)}{2 k-1}
$$

Then the sequence $\left\{\frac{b_{n}}{n !}\right\}_{n \geq 0}$ satisfies the higher order Turán inequality.

Proof. Use Zeilberger's algorithm we see that $\left\{b_{n}\right\}_{n \geq 0}$ satisfies the following recurrence relation

$$
(n+3) b_{n+3}-(7 n+13) b_{n+2}+(7 n+15) b_{n+1}-(n+1) b_{n}=0, \quad n \geq 0,
$$

with the initial values

$$
b_{0}=-1, \quad b_{1}=1, \quad b_{2}=7 .
$$

By the algorithm HT, we have the following estimates:

$$
1+\left(\frac{3(17+12 \sqrt{2})}{2(3+2 \sqrt{2})^{2}}-1\right) \frac{1}{n^{2}}<\frac{b_{n+1} b_{n-1}}{b_{n}^{2}}<1+\left(\frac{3(17+12 \sqrt{2})}{2(3+2 \sqrt{2})^{2}}+1\right) \frac{1}{n^{2}}, \quad n \geq 16,
$$


implying that

$$
1<\frac{b_{n+1} b_{n-1}}{b_{n}^{2}}<1+\frac{3}{n^{2}}, \quad n \geq 16
$$

Let

$$
a_{n}=\frac{b_{n}}{n !}, \quad u_{n}=\frac{a_{n+1} a_{n-1}}{a_{n}^{2}} .
$$

Then we have

$$
\frac{n}{n+1}<u_{n}<\frac{n}{n+1}\left(1+\frac{3}{n^{2}}\right), \quad n \geq 16 .
$$

Let

$$
g_{n}=\frac{n}{n+1}, \quad \text { and } \quad f_{n}=\frac{n}{n+1}\left(1+\frac{3}{n^{2}}\right) .
$$

Direct computation shows that

$$
t\left(g_{n}, g_{n+1}\right)=\frac{4}{(n+1)(n+2)^{2}},
$$

which is positive for $n \geq 0$.

Similarly, we have

$$
\begin{gathered}
t\left(g_{n}, f_{n+1}\right)=\frac{4 n^{3}-21 n^{2}-36 n-20}{(n+1)^{4}(n+2)^{2}}>0, \quad n>6 . \\
t\left(f_{n}, g_{n+1}\right)=\frac{4 n^{2}-21 n-9}{n^{2}(n+1)(n+2)^{2}}>0, \quad n>5 . \\
t\left(f_{n}, f_{n+1}\right)=\frac{4 n^{5}-24 n^{4}+36 n^{3}+16 n^{2}-48 n-144}{n^{2}(n+1)^{4}(n+2)^{2}}>0, \quad n>7 .
\end{gathered}
$$

Checking the initial values, we finally get $\left\{\frac{b_{n}}{n !}\right\}_{n \geq 0}$ satisfies the higher order Turán inequality.

Acknowledgments. We would like to thank the referees for their helpful comments. This work was supported by the National Natural Science Foundation of China (grants 11771330 and 11921001).

\section{References}

[1] G. D. Birkhoff and W. J. Trjitzinsky, Analytic theory of singular difference equations, Acta Math., 60(1) (1933) 1-89.

[2] W.Y.C. Chen, D.X.Q. Jia, L.X.W. Wang. Higher order Turán inequalities for the partition function, Trans. Amer. Math. Soc., 372(3) (2019) 2143-2165. 
[3] G. Csordas, Complex zero decreasing sequences and the Riemann hypothesis II, In: Analysis and Applications — ISAAC 2001, Springer, Boston, MA, 2003, pp. 121-134.

[4] S. DeSalvo and I. Pak, Log-concavity of the partition function, Ramanujan J., 38(1) (2014) 1-13.

[5] D. K. Dimitrov, Higher Order Turán Inequalities, Proc. Amer. Math. Soc., 126(7) (1998) 2033-2037.

[6] T. Došlić, Log-balanced for combinatorial sequences, Int. J. Math. Sci., 2005 (2005) 507-522.

[7] M. Griffin, K. Ono, L. Rolen, D. Zagier, Jensen polynomials for the Riemann zeta function and other sequences, Proc. Natl. Acad. Sci. USA, 116(23) (2019) 11103-11110.

[8] Q. H. Hou and Z. R. Zhang, Asymptotic $r$-log-convexity and P-recursive sequences, J. Symbolic Comput., 93(2018) 21-33.

[9] M. Kauers, A Mathematica package for computing asymptotic expansions of solutions of $P$-finite recurrence equations, Technical Report RISC 11-04, 2011.

[10] P. R. W. McNamara and B. E. Sagan, Infinite log-concavity: Developments and conjectures, Adv. in Appl. Math., 44(1) (2010) 1-15.

[11] V. H. Moll, Combinatorial sequences arising from a rational integral, Online J. Anal. Combin. 2 (2007) \#4.

[12] C. P. Niculescu, A new look at Newton's inequalities, J. Inequal. Appl., 1(2)2013.

[13] R. P. Stanley, Log-concave and unimodal sequences in algebra, combinatorics, and geometry, Ann. New York Acad. Sci., 576(1) (1989) 500-535.

[14] R. P. Stanley, Enumerative Combinatorics, Volume 2, Cambridge University Press, Cambridge,(1999).

[15] G. Szegő, On an inequality of P. Turán concerning Legendre polynomials, Bull. Amer. Math. Soc., 54 (1948) .

[16] L. X. W. Wang, Higher order Turán inequalities for combinatorial sequences, Adv. in Appl. Math., 110 (2019) 180-196.

[17] C. G. Wagner, Newton's inequality and a test for imaginary roots, Two-Year College Math. J., 8(3) (1977) 145-147.

[18] J. Wimp and D. Zeilberger, Resurrecting the asymptotics of linear recurrences, J. Math. Anal. Appl., 111 (1985) 162-176.

[19] D. Zeilberger, Asyrec: a Maple package for computing the asymptotics of solutions of linear recurrence equations with polynomial coefficients, 2016.

[20] http://faculty.tju.edu.cn/HouQinghu/en/index.htm 